\title{
Structural and Electronic Properties of Nitrogen-Doped Graphene
}

\author{
J. Sforzini, ${ }^{1,2}$ P. Hapala, ${ }^{3}$ M. Franke, ${ }^{1,2}$ G. van Straaten, ${ }^{1,2}$ A. Stöhr,${ }^{4}$ S. Link, ${ }^{4}$ S. Soubatch, ${ }^{1,2}$ P. Jelínek, ${ }^{3}$ \\ T.-L. Lee, ${ }^{5}$ U. Starke, ${ }^{4}$ M. Švec, ${ }^{3}$ F. C. Bocquet, ${ }^{1,2, *}$ and F. S. Tautz ${ }^{1,2}$ \\ ${ }^{1}$ Peter Grünberg Institut (PGI-3), Forschungszentrum Jülich, 52425 Jülich, Germany \\ ${ }^{2}$ Jülich Aachen Research Alliance (JARA), Fundamentals of Future Information Technology, 52425 Jülich, Germany \\ ${ }^{3}$ Institute of Physics of the Czech Academy of Sciences, Cukrovarnická 10, 16200 Prague, Czech Republic \\ ${ }^{4}$ Max Planck Institute for Solid State Research, Heisenbergstraße, 70569 Stuttgart, Germany \\ ${ }^{5}$ Diamond Light Source Ltd, Didcot OX110DE, Oxfordshire, United Kingdom
}

(Received 17 July 2015; revised manuscript received 22 January 2016; published 24 March 2016)

\begin{abstract}
We investigate the structural and electronic properties of nitrogen-doped epitaxial monolayer graphene and quasifreestanding monolayer graphene on $6 H$-SiC $(0001)$ by the normal incidence x-ray standing wave technique and by angle-resolved photoelectron spectroscopy supported by density functional theory simulations. With the location of various nitrogen species uniquely identified, we observe that for the same doping procedure, the graphene support, consisting of substrate and interface, strongly influences the structural as well as the electronic properties of the resulting doped graphene layer. Compared to epitaxial graphene, quasifreestanding graphene is found to contain fewer nitrogen dopants. However, this lack of dopants is compensated by the proximity of nitrogen atoms at the interface that yield a similar number of charge carriers in graphene.
\end{abstract}

DOI: 10.1103/PhysRevLett.116.126805

The discovery of graphene and its exceptional properties has triggered very active research on this material $[1,2]$. One strong focus has been aiming at modifying graphene's electronic properties for device applications [3]. Substituting the carbon atoms (C) by other elements is one way to dope the layer. To date, nitrogen $(\mathrm{N})$ belongs to the most studied dopants for graphene [4-6]. This is demonstrated by the remarkable performance improvements resulting from the use of $\mathrm{N}$-doped graphene-based components in, e.g., lithium-ion batteries [7], ultracapacitors [8,9], fuel cells [10], and field-effect transistors $[11,12]$. However, our understanding of the doping process at the atomic level is still in its infancy. It is suspected that $\mathrm{N}$ incorporation alters the structure of graphene and degrades its quality [13,14], while other factors, such as the effects of the underlying support (i.e., the substrate and possibly an interface layer) on the doping, remain largely unexplored.

In this Letter, we study the effects of $\mathrm{N}$ doping on the structural and electronic properties of graphene, with particular emphasis on the influence of the support on the doping. To this end, N-doped epitaxial monolayer graphene (EMLG) and H-intercalated quasifreestanding monolayer graphene (QFMLG), both grown on $6 H$-SiC $(0001)$, are investigated by the normal incidence $\mathrm{x}$-ray standing wave (NIXSW) technique, which determines the vertical positions of the individual chemical

Published by the American Physical Society under the terms of the Creative Commons Attribution 3.0 License. Further distribution of this work must maintain attribution to the author $(s)$ and the published article's title, journal citation, and DOI. species in the near surface region. The effects of the structural changes on the electronic bands are checked by angle-resolved photoelectron spectroscopy (ARPES) and density functional theory (DFT). We find that the support dramatically influences how much $\mathrm{N}$, as well as how $\mathrm{N}$, incorporates in graphene, which in turn affects the dopant-induced band broadening. In the case of EMLG, the support itself remains structurally intact after the doping. In contrast, the QFMLG/SiC interface becomes $\mathrm{N}$ doped, and consequently graphene is pushed locally by the $\mathrm{N}$ atoms away from the $\mathrm{H}$ terminated $\mathrm{SiC}$ substrate and buckles. Furthermore, the interaction with the thus doped interface provides additional charge carriers to graphene in QFMLG.

The samples were prepared following the procedure described in Ref. [15]. The in situ $\mathrm{N}$ doping and the x-ray measurements were performed at the I09 beam line, Diamond Light Source [16]. Before doping, the absence of $\mathrm{N}$ from the sample surfaces was confirmed by $\mathrm{x}$-ray photoelectron spectroscopy (XPS). The EMLG and QFMLG were doped by exposing the samples (at room temperature) to $100 \mathrm{eV} \mathrm{N}^{+}$ions for $4 \mathrm{~min}$ and annealing at 1200 and $1000 \mathrm{~K}$, respectively. The $\mathrm{N}_{2}$ pressure during the ion implantation did not exceed $6 \times 10^{-7}$ mbar. The absence of point defects induced by this implantation method has been checked by scanning tunneling microscopy (STM) for annealing temperatures as low as $1000 \mathrm{~K}$. Furthermore, we confirmed the stability of the $\mathrm{H}$ intercalation layer at $1000 \mathrm{~K}$ in QFMLG using ARPES. Higher doping levels were achieved for EMLG and QFMLG by repeating this procedure. A sample with only a buffer layer (BL) was also studied as a reference. 
We first present the structures of N-doped graphene as determined by the NIXSW technique. We measured a set of XPS spectra (C $1 s, \mathrm{Si} 2 p, \mathrm{~N} 1 s)$ over a photon energy range of $2 \mathrm{eV}$ around the (0006) Bragg energy (2462 eV) of $6 \mathrm{H}$-SiC. Fitting the photoelectron yield (EY) as a function of the photon energy with theory [17] determines two parameters for each chemical component of a core level: the coherent position $P^{H}$ and the coherent fraction $F^{H}$, which represent the position of the emitter between two Bragg planes and its spread perpendicular to the planes, respectively. Knowing the Bragg plane spacing of the $H=(0006)$ reflection of the $6 H-\mathrm{SiC}$ crystal $\left(d_{H}=2.517 \AA\right)$, the possible vertical positions $z_{X}$ of each component $X$ with respect to the topmost $\mathrm{Si}$ layer can be calculated by $z_{X}=\left(n+P_{X}^{H}-P_{\mathrm{Si}}^{H}\right) \times d_{H}$, where $n$ is an integer, $P_{X}^{H}$ and $P_{\mathrm{Si}}^{H}$ are the coherent positions of the component $X$ and of the top few Si layers, respectively. The results for $\mathrm{Si} 2 p$ and C $1 s$ of the undoped samples, neglecting nondipolar effects [18] and summarized in Table I, are in excellent agreement with the literature [19].

In Figs. 1(a)-1(c), N $1 s$ spectra are shown for the BL, EMLG, and QFMLG samples after 4 min of doping. The spectra of the three samples present different sets of components, revealing the distinct chemical states of $\mathrm{N}$. The EY curves of the different $\mathrm{N} 1 s$ components together with their best fits are displayed in Figs. 1(d)-1(f). Using the vertical positions derived from the NIXSW technique, summarized in Table I, we will show below that these chemical components can be associated with $\mathrm{N}$ incorporation into the different constituents of the samples.

Before considering the results for EMLG and QFMLG, it is worth studying the effect of $\mathrm{N}$ doping on the BL sample, because the buffer layer on $\mathrm{SiC}$ is also the support for graphene in EMLG. The $\mathrm{N} 1 s$ spectrum of the BL [Fig. 1(a)] exhibits a main component at $397.5 \mathrm{eV}$ and a shoulder at $399.7 \mathrm{eV}$. The main component is also present for the EMLG and QFMLG samples [Figs. 1(b) and 1(c)]. The NIXSW analysis of this component for the three doped samples renders a common coherent position $P_{\mathrm{N}_{\mathrm{SiC}}}^{H}$ of $0.66 \pm 0.01$ [Figs. 1(d)-1(f)], which does not match the vertical positions of any of the $\mathrm{C}$ or intercalation layers. As only the $\mathrm{SiC}$ substrate is common to all three samples, and since the binding energy of $397.5 \mathrm{eV}$ agrees with the previous measurements on $\mathrm{N}$-doped bulk $\mathrm{SiC}$ [22], we assign this $\mathrm{N} 1 s$ component to $\mathrm{N}$ atoms that have diffused into the substrate during annealing, or that were directly implanted into the bulk during sputtering, designated as $\mathrm{N}_{\mathrm{SiC}}$ in the following. $\mathrm{N}_{\mathrm{SiC}}$ is found to be $0.21 \pm 0.09 \AA$ below the $\mathrm{C}$ planes in the bulk $\left(\mathrm{C}_{\mathrm{SiC}}\right)$. A further interpretation of its exact position is beyond the scope of this Letter. Besides the common component $\mathrm{N}_{\mathrm{SiC}}$, the spectrum of the doped $\mathrm{BL}$ sample exhibits a shoulder at a binding energy of $399.7 \mathrm{eV}$ [Fig. 1(a)], which has also been observed in heavily doped $\mathrm{SiC}$ [22]. Moreover, it is found that the BL vertical position $\left(z_{\mathrm{C}_{\mathrm{BL}}}=2.30 \pm 0.02 \AA\right.$ before and $2.28 \pm 0.02 \AA$ after doping) and its buckling $\left(F_{\mathrm{C}_{\mathrm{BL}}}^{H}=0.77 \pm 0.04\right.$ to $\left.0.74 \pm 0.04\right)$ are hardly affected by the doping. This suggests that $\mathrm{N}$ dopants are unstable in the BL and tend to diffuse into the $\mathrm{SiC}$ substrate upon annealing, leaving the BL intact.

The $\mathrm{N} 1 s$ spectra for both EMLG and QFMLG [Figs. 1(b) and 1(c)] show features at binding energies between 3 and $5.5 \mathrm{eV}$ above $\mathrm{N}_{\mathrm{SiC}}$. A closer look at Fig. 1(b) for EMLG reveals that more than one component is necessary to fit this peak. We present a fit result with three components at 400.7, 401.8, and $403.1 \mathrm{eV}$. Although their chemical states are different, the three $\mathrm{N}$ species share the same vertical position $P_{\mathrm{N}_{\mathrm{gr}}}^{H}=0.31$ (also when assuming a two-component model) and a high degree of ordering along the vertical direction, $F_{\mathrm{N}_{\mathrm{gr}}}^{H}=0.76 \pm 0.17$ (Table I). The sum of the three components is represented by the bottom EY curve in Fig. 1(e). Converting $P_{\mathrm{N}_{\text {or }}}^{H}$ to

TABLE I. Summary of NIXSW results before and after 4 min of doping. For $\mathrm{C}_{\mathrm{SiC}}$ and $\mathrm{N}_{\mathrm{SiC}}$, the $z$ value is given for $n=-1$. The error bar on $F^{H}$ is 0.04 , and 0.17 for $\mathrm{C} 1 s$, and $\mathrm{N} 1 s$ components.

\begin{tabular}{|c|c|c|c|c|c|c|c|c|c|c|}
\hline \multirow[t]{2}{*}{ Pristine } & \multicolumn{3}{|c|}{ BL } & \multicolumn{3}{|c|}{ EMLG } & \multicolumn{3}{|c|}{ QFMLG } & \multirow[b]{2}{*}{ Error $(\AA)$} \\
\hline & $P^{H}$ & $F^{H}$ & $z(\AA)$ & $P^{H}$ & $F^{H}$ & $z(\AA)$ & $P^{H}$ & $F^{H}$ & $z(\AA)$ & \\
\hline $\mathrm{Si}$ & 0.03 & 1.31 & 0 & 0.04 & 1.33 & 0 & 0.03 & 1.31 & 0 & \\
\hline $\mathrm{C}_{\mathrm{SiC}}$ & 0.75 & 1.10 & -0.70 & 0.76 & 1.04 & -0.68 & 0.75 & 0.99 & -0.71 & \pm 0.01 \\
\hline $\mathrm{C}_{\mathrm{BL}}$ & 0.94 & 0.77 & 2.30 & 0.97 & 0.53 & 2.37 & & & & \pm 0.02 \\
\hline $\mathrm{C}_{\mathrm{gr}}$ & & & & 0.29 & 1.01 & 5.67 & 0.73 & 0.68 & 4.28 & \pm 0.01 \\
\hline Doped & & BL & & & EMLC & & & QFML & & \\
\hline $\mathrm{Si}$ & 0.04 & 1.33 & 0 & 0.03 & 1.31 & 0 & 0.03 & 1.31 & 0 & \\
\hline $\mathrm{C}_{\mathrm{SiC}}$ & 0.76 & 1.07 & -0.70 & 0.76 & 1.03 & -0.69 & 0.76 & 1.01 & -0.70 & \pm 0.01 \\
\hline $\mathrm{C}_{\mathrm{BL}}$ & 0.94 & 0.74 & 2.28 & 0.98 & 0.41 & 2.37 & & & & \pm 0.02 \\
\hline $\mathrm{C}_{\mathrm{gr}}$ & & & & 0.28 & 1.13 & 5.65 & 0.84 & 0.52 & 4.56 & \pm 0.01 \\
\hline $\mathrm{N}_{\mathrm{SiC}}$ & 0.67 & 0.97 & -0.91 & 0.66 & 0.79 & -0.94 & 0.65 & 0.29 & -0.96 & \pm 0.09 \\
\hline $\mathrm{N}_{\mathrm{gr}}$ & & & & 0.31 & 0.76 & 5.72 & 0.82 & 0.75 & 4.49 & \pm 0.05 \\
\hline $\mathrm{N}_{\text {int }}$ & & & & & & & 0.11 & 0.98 & 2.71 & \pm 0.04 \\
\hline
\end{tabular}



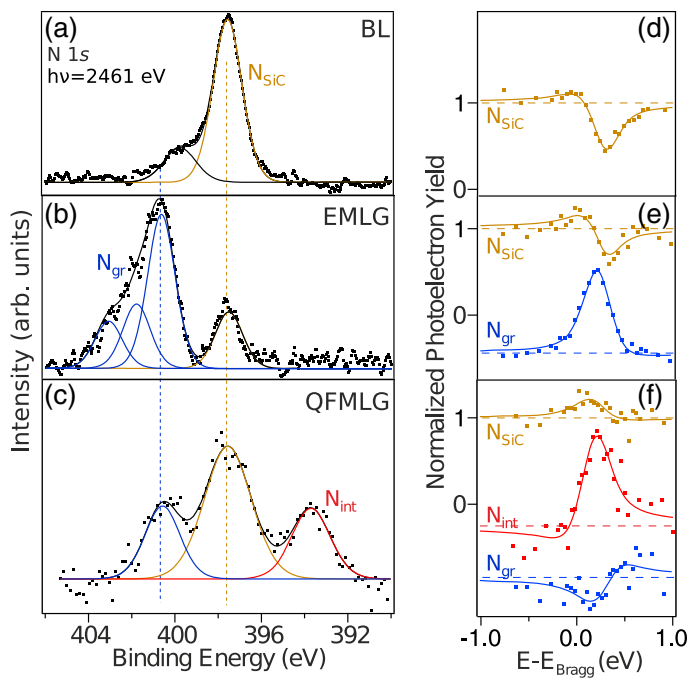

FIG. 1. $\mathrm{N}$-doped samples after $4 \mathrm{~min}$ of $\mathrm{N}$ ion bombardment and annealing. N $1 s$ XPS spectra for (a) the BL, (b) the EMLG, and (c) the QFMLG. The spectra were fitted using symmetrical Voigt line shapes. The experimental data points are shown as black dots, and the envelope as a black line. $\mathrm{N}_{\mathrm{SiC}}$ is displayed in orange, $\mathrm{N}_{\mathrm{gr}}$ in blue, and $\mathrm{N}_{\text {int }}$ in red. The corresponding EY curves are displayed in panels (d)-(f) with their best fits. The EY curves of $\mathrm{N}_{\mathrm{gr}}$ and $\mathrm{N}_{\mathrm{int}}$ are offset for clarity.

$z_{\mathrm{N}_{\mathrm{gr}}}$, we find that with $n=2$ the height of the three species $\left(z_{\mathrm{N}_{\mathrm{gr}}}=5.72 \pm 0.05 \AA\right)$ corresponds to the graphene vertical position [see Fig. 2(a)], which is not affected by the doping $\left(z_{\mathrm{C}_{\mathrm{gr}}}=5.67 \pm 0.01 \AA\right.$ before and $5.65 \pm 0.01 \AA$ after $\mathrm{N}$ doping). We therefore attribute the three $\mathrm{N} 1 s$ components to $\mathrm{N}$ atoms incorporated into the graphene layer $\left(\mathrm{N}_{\mathrm{gr}}\right)$ of EMLG. The NIXSW analysis of the BL in the EMLG sample shows that it remains unperturbed after $\mathrm{N}$ implantation [see Table I and Fig. 2(a)], as also observed above for the BL sample. Therefore, doping the EMLG with $\mathrm{N}$ does not affect the overlap of the van der Waals (vdW) radii or the interaction between the graphene layer and its support.

We note that a recent combined XPS and STM study [23] reported a nearly identical $\mathrm{N} 1 s$ line shape as observed here for a N-doped mixture of mono- and bilayer graphene on $6 \mathrm{H}$-SiC, where $\mathrm{N}_{\mathrm{gr}}$ was modeled with two components associated with the graphene multilayer. This peak assignment is incompatible with the present NIXSW analysis, which shows that all $\mathrm{N}_{\mathrm{gr}}$ components share the same vertical position. However, a key observation offering a plausible interpretation of the line shape of $\mathrm{N}_{\mathrm{gr}}$ comes from the STM results in Ref. [23], which reveal a mixture of single $\mathrm{N}$ atoms and aggregates of two $\mathrm{N}$ atoms (mostly in a metaconfiguration) for the doping concentrations relevant to the present XPS and NIXSW experiments. We therefore assign the multiple $\mathrm{N}_{\mathrm{gr}}$ components to single $\mathrm{N}$ atoms as well as to aggregates of two or more $\mathrm{N}$ atoms in graphene.

We now turn to QFMLG. In contrast to EMLG, after doping QFMLG, its graphene layer is pushed up significantly from $z_{\mathrm{C}_{\mathrm{gr}}}=4.28 \pm 0.01 \AA$ to $4.56 \pm 0.01 \AA$

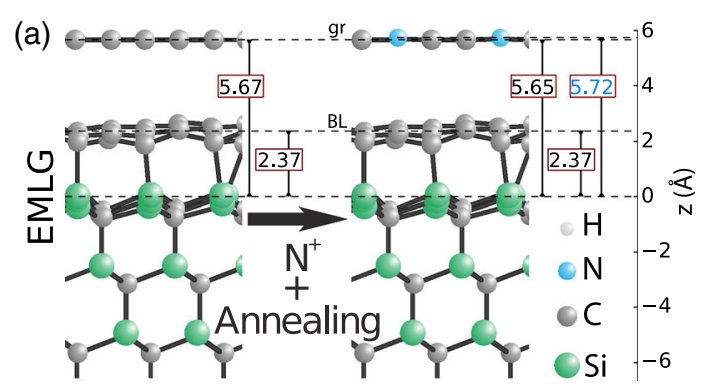

(b)

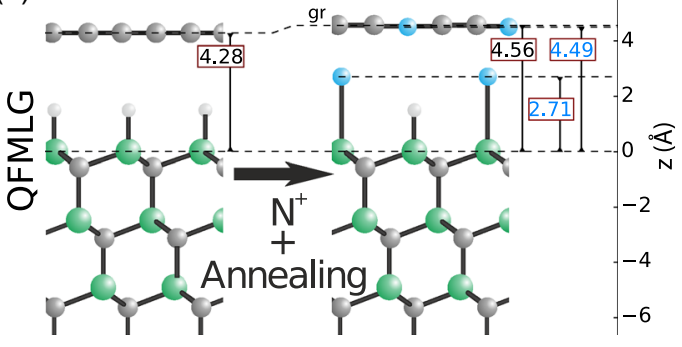

FIG. 2. Summary of the vertical positions (in $\AA$ ) measured by the NIXSW technique for each atomic species in (a) EMLG and in (b) QFMLG, before and after N doping.

[see Table I and Fig. 2(b)]. The analysis of the EY curve for the $\mathrm{N} 1 s$ component at $400.6 \mathrm{eV}$ [Fig. 1(c)] yields a vertical position of $z_{\mathrm{N}_{\mathrm{gr}}}=4.49 \pm 0.05 \AA$ that is comparable to $z_{\mathrm{C}_{\mathrm{gr}}}$ of the doped graphene layer. One can therefore associate this component with the $\mathrm{N}$ implanted in graphene $\left(\mathrm{N}_{\mathrm{gr}}\right)$. However, unlike doped EMLG, where multiple $\mathrm{N}_{\mathrm{gr}}$ components are present [Fig. 1(b)], $\mathrm{N}_{\mathrm{gr}}$ of doped QFMLG represents a single type of dopant, as evidenced by the well-defined $\mathrm{N} 1 s$ component at $400.6 \mathrm{eV}$, which has the same binding energy as the main component of $\mathrm{N}_{\mathrm{gr}}$ in EMLG (indicating that this dopant configuration is least dependent on the choice of support). Furthermore, by comparing the intensity of $\mathrm{N}_{\mathrm{gr}}$, normalized to the intensity of the graphene component in the $\mathrm{C} 1 s$ core level, we find that there are twice less $\mathrm{N}$ atoms substituting $\mathrm{C}$ in the graphene honeycomb lattice of QFMLG than in that of EMLG, for the same $\mathrm{N}$ sputter conditions.

In addition to $\mathrm{N}_{\mathrm{SiC}}$ and $\mathrm{N}_{\mathrm{gr}}$, doped QFMLG exhibits a third $\mathrm{N} 1 s$ component at a binding energy of $393.7 \mathrm{eV}$ [Fig. 1(c)]. Our NIXSW analysis shows that this component $\mathrm{N}_{\text {int }}$, which yields a vertical position $z_{\mathrm{N}_{\text {int }}}=2.71 \pm 0.04 \AA$, can be best interpreted as $\mathrm{N}$ atoms replacing some of the $\mathrm{H}$ atoms within the intercalation layer. Because of its larger atomic size, $\mathrm{N}_{\text {int }}$ is situated at a higher position than that expected for $\mathrm{H}\left(z_{\mathrm{H}}=1.5 \AA\right)$ [24], leading to the abovementioned elevation of graphene after the doping. Note, however, that graphene is lifted less $(0.28 \AA)$ than the $\mathrm{H}$ to $\mathrm{N}$ height difference $(1.21 \AA)$. This means that locally the vdW radii of the doped graphene layer and the intercalated $\mathrm{N}$ atoms must overlap. As we will see below, this has an important consequence for the electronic properties of $\mathrm{N}$ doped QFMLG. Also note that the concomitant reduction of 
the graphene coherent fraction from $F_{\mathrm{C}_{\mathrm{gr}}}^{H}=0.68 \pm 0.04$ to $0.52 \pm 0.04$ upon doping is an indication that the graphene is now buckled. Our result thus shows that, while pristine QFMLG exhibits the weakest coupling with the substrate [24], its structure is more affected by N doping than that of EMLG. We stress that considering the low concentration of $\mathrm{N}$ and the stability of the intercalated $\mathrm{H}$ at $1000 \mathrm{~K}$, as proven by the presence of the Dirac cone after annealing [Figs. 3(e) and 3(f)], a scenario in which $\mathrm{H}$ is either fully desorbed or fully replaced by $\mathrm{N}$ can be excluded.

Having established the structural modifications of graphene under $\mathrm{N}$ doping, we now discuss the effects on its electronic properties. ARPES spectra measured around the $\bar{K}$ point of EMLG and QFMLG are shown in Fig. 3 for pristine graphene and for two doping steps. As expected, the Dirac point of the pristine EMLG is found about $0.4 \mathrm{eV}$ below the Fermi edge [15]. The first doping step of $4 \mathrm{~min}$ shifts the Dirac cone towards higher binding energies by $0.2 \mathrm{eV}$, which corresponds to a carrier density increase of $1.4 \times 10^{13} \mathrm{~cm}^{-2}$ [25]. It thus confirms that the $\mathrm{N}$ incorporation into the graphene sheet has led to $n$-type doping. Further doping continues to increase the carrier density, but at a lower rate: $0.9 \times 10^{13} \mathrm{~cm}^{-2}$ over the next 4 min of doping [Fig. 3(c)]. QFMLG presents similar results: $1.2 \times 10^{13} \mathrm{~cm}^{-2}$ and $0.6 \times 10^{13} \mathrm{~cm}^{-2}$ increases for the first and second doping steps, respectively. Besides doping graphene, the introduction of dopants in the lattice also induces a broadening of the $\pi$ band after each doping step. We note, however, that this broadening is more pronounced for

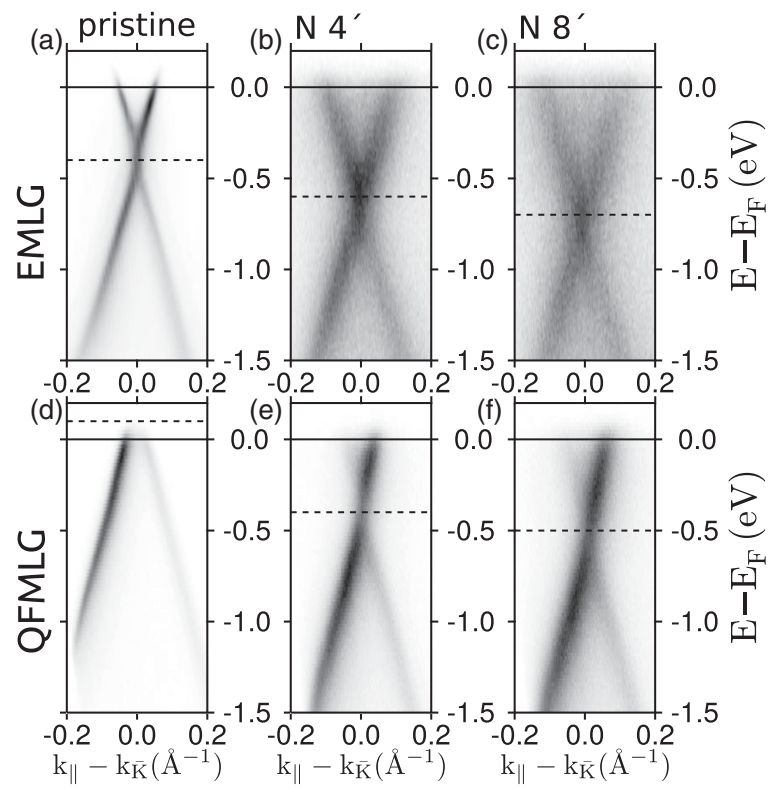

FIG. 3. ARPES spectra of the EMLG (a)-(c) and QFMLG (d)-(f) valence measured around the $\overline{\mathrm{K}}$ point in the $\bar{\Gamma} \overline{\mathrm{K}}$ direction of the graphene Brillouin zone with a $\mathrm{HeI}_{\alpha}$ radiation for (a) and (d) pristine graphene, (b) and (e) after $4 \mathrm{~min}$, (c) and (f) after 8 min doping. The Fermi and Dirac energies are represented by dashed and solid lines, respectively. In panels (d)-(f) and (a)-(c) the UV lamp is installed in and out of the scattering plane, respectively.
EMLG than for QFMLG. As demonstrated in Ref. [23] and confirmed by STM in the present work for surfaces annealed to $1000 \mathrm{~K}$ (not shown), the method used here for doping does not introduce any defects in the graphene lattice other than substitutional $\mathrm{N}$ dopants. Therefore, the band broadening cannot stem from a defective lattice.

To better understand the origin of this broadening, we simulated the effects of $\mathrm{N}$ doping on the band structure of graphene. We performed total energy DFT [26] simulations on a $(12 \times 12)$ supercell of freestanding graphene containing three randomly distributed $\mathrm{N}$ substitutional dopants in the lattice. Four different dopant configurations have been included, and one example is given in Fig. 4(c). After relaxing the geometric structure, the band structure of the supercell is projected onto the first Brillouin zone for a direct comparison with the experimental data [27]. The results are shown in Fig. 4. Each of the four dopant configurations yields a slightly modified band structure with a band gap smaller than $0.1 \mathrm{eV}$ and a slightly different downward energy shift of about $0.2 \mathrm{eV}$ with respect to the pristine graphene. Superimposing them results in an effective broadening of the $\pi$ band of graphene, without opening a sizable band gap. We therefore attribute the band broadening observed in the experimental ARPES data shown in Fig. 3 to the random $\mathrm{N}$ substitution in the graphene lattice.

From our combined study, an understanding of how the various $\mathrm{N}$ species contribute to the doping of graphene can be reached: For the same sputtering conditions, XPS reveals that the total amount of $\mathrm{N}$ incorporated in the graphene lattice $\left(\mathrm{N}_{\mathrm{gr}}\right)$ of QFMLG is only half of that of EMLG, while ARPES shows comparable increases of carrier densities for the two samples. This indicates that $\mathrm{N}$ atoms substituting hydrogen at the interface of QFMLG $\left(\mathrm{N}_{\text {int }}\right)$ also contribute to the doping. This is consistent with the strong overlap of vdW radii between graphene and $\mathrm{N}_{\mathrm{int}}$ that is observed by NIXSW for QFMLG. In contrast to EMLG, where $\mathrm{N}_{\mathrm{gr}}$ contains multiple components but no $\mathrm{N}$ exists in the BL, the additional doping induced by $\mathrm{N}_{\text {int }}$ in QFMLG allows the same

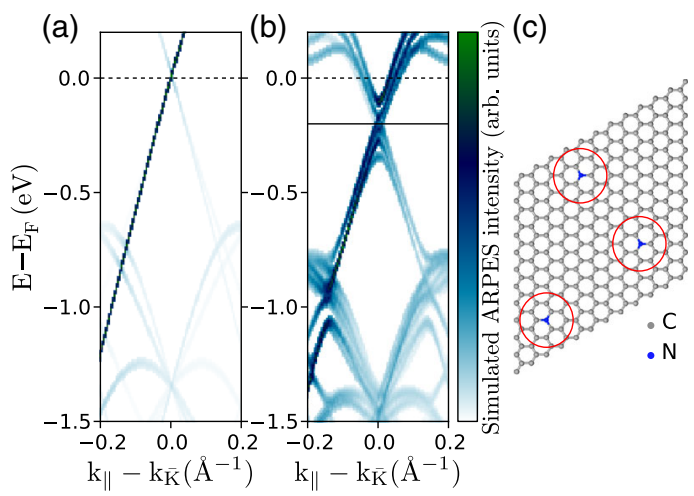

FIG. 4. (a) Simulated ARPES spectrum of a freestanding graphene layer, relaxed in a $12 \times 12$ supercell. (b) Superimposition of four simulated ARPES spectra of differently N-doped freestanding graphene supercells. (c) Sketch of one of the N-doped supercells used in (b). The $\mathrm{N}$ atoms are highlighted by red circles. 
number of charge carriers to be generated, albeit with fewer substitutional dopants in graphene that are, moreover, of a single type only, both of which in turn reduce the $\pi$-band broadening of QFMLG in ARPES.

To conclude, we find clear evidence that the support has multiple influences on the doping of a graphene layer. It affects the quantity as well as the variety of dopants created in the honeycomb lattice. Furthermore, we show that the support can host foreign species that make an additional contribution to the doping of graphene.

F.C. B. acknowledges financial support from the Initiative and Networking Fund of the Helmholtz Association, Postdoc Programme VH-PD-025. Support by the German Research Foundation (DFG) in the framework of the Priority Program 1459 Graphene, project Sta315/8-2 is gratefully acknowledged. This work has been supported by the grant agency of the Czech republic, Grant No. 15-07172S. Access to computing and storage facilities owned by parties and projects contributing to the Czech National Grid Infrastructure MetaCentrum, provided under the program "Projects of Large Infrastructure for Research, Development, and Innovations" (LM2010005), is greatly appreciated. The authors would like to thank D. McCue for his excellent technical support at the I09 beam line.

*f.bocquet@fz-juelich.de

[1] A. K. Geim, Graphene: Status and prospects, Science 324, 1530 (2009).

[2] A. H. Castro Neto, F. Guinea, N. M. R. Peres, K. S. Novoselov, and A. K. Geim, The electronic properties of graphene, Rev. Mod. Phys. 81, 109 (2009).

[3] Q. Tang, Z. Zhou, and Z. Chen, Graphene-related nanomaterials: tuning properties by functionalization, Nanoscale 5, 4541 (2013).

[4] L. S. Panchakarla, K. S. Subrahmanyam, S. K. Saha, A. Govindaraj, H. R. Krishnamurthy, U. V. Waghmare, and C. N. R. Rao, Synthesis, structure, and properties of boronand nitrogen-doped graphene, Adv. Mater. 21, 4726 (2009).

[5] F. Karlický, K. Kumara Ramanatha Datta, M. Otyepka, and R. Zbořil, Halogenated graphenes: Rapidly growing family of graphene derivatives, ACS Nano 7, 6434 (2013).

[6] H. Wang, T. Maiyalagan, and X. Wang, Review on recent progress in nitrogen-doped graphene: Synthesis, characterization, and its potential applications, ACS Catal. 2, 781 (2012).

[7] A. L. M. Reddy, A. Srivastava, S. R. Gowda, H. Gullapalli, M. Dubey, and P. M. Ajayan, Synthesis of nitrogen-doped graphene films for lithium battery application, ACS Nano 4, 6337 (2010).

[8] H. M. Jeong, J. W. Lee, W. H. Shin, Y. J. Choi, H. J. Shin, J. K. Kang, and J. W. Choi, Nitrogen-doped graphene for high-performance ultracapacitors and the importance of nitrogen-doped sites at basal planes, Nano Lett. 11, 2472 (2011).

[9] M. D. Stoller, S. Park, Y. Zhu, J. An, and R. S. Ruoff, Graphene-based ultracapacitors, Nano Lett. 8, 3498 (2008).
[10] B. Xia, Y. Yan, X. Wang, and X. W. Lou, Recent progress on graphene-based hybrid electrocatalysts, Mater. Horiz. 1, 379 (2014).

[11] D. Wei, Y. Liu, Y. Wang, H. Zhang, L. Huang, and G. Yu, Synthesis of N-doped graphene by chemical vapor deposition and its electrical properties, Nano Lett. 9, 1752 (2009).

[12] B. Guo, Q. Liu, E. Chen, H. Zhu, L. Fang, and J.-R. Gong, Controllable N-doping of graphene, Nano Lett. 10, 4975 (2010).

[13] D. Geng, S. Yang, Y. Zhang, J. Yang, J. Liu, R. Li, T.-K. Sham, X. Sun, S. Ye, and S. Knights, Nitrogen doping effects on the structure of graphene, Appl. Surf. Sci. 257, 9193 (2011).

[14] D. Y. Usachov, A. V. Fedorov, O. Y. Vilkov, B. V. Senkovskiy, V. K. Adamchuk, B. V. Andryushechkin, and D. V. Vyalikh, Synthesis and electronic structure of nitrogen-doped graphene, Phys. Solid State 55, 1325 (2013).

[15] C. Riedl, C. Coletti, and U. Starke, Structural and electronic properties of epitaxial graphene on $\mathrm{SiC}(0001)$ : A review of growth, characterization, transfer doping and hydrogen intercalation, J. Phys. D 43, 374009 (2010).

[16] More details of the experiments and the end-station can be found in Ref. [24].

[17] D. P. Woodruff, Surface structure determination using X-ray standing waves, Rep. Prog. Phys. 68, 743 (2005).

[18] D. P. Woodruff, Non-dipole effects in high-energy photoelectron emission; identification and quantification using X-ray standing waves, Nucl. Instrum. Methods Phys. Res., Sect. A 547, 187 (2005).

[19] See Refs. [20,21,24]. As an example, the measured positions $\mathrm{z}_{\mathrm{C}_{\mathrm{BL}}}$ of the $\mathrm{C}$ atoms in the buffer layer relative to the topmost $\mathrm{Si}$ atoms are $2.30 \pm 0.02 \AA$ and $2.37 \pm 0.02 \AA$ for the BL and EMLG samples, respectively, compared to predicted positions of $2.32 \AA$ and $2.36 \AA$ [20].

[20] L. Nemec, V. Blum, P. Rinke, and M. Scheffler, Thermodynamic Equilibrium Conditions of Graphene Films on SiC, Phys. Rev. Lett. 111, 065502 (2013).

[21] J. D. Emery, B. Detlefs, H. J. Karmel, L. O. Nyakiti, D. K. Gaskill, M. C. Hersam, J. Zegenhagen, and M. J. Bedzyk, Chemically Resolved Interface Structure of Epitaxial Graphene on SiC(0001), Phys. Rev. Lett. 111, 215501 (2013).

[22] M. B. Wijesundara, C. R. Stoldt, C. Carraro, R. T. Howe, and R. Maboudian, Nitrogen doping of polycrystalline 3C-SiC films grown by single-source chemical vapor deposition, Thin Solid Films 419, 69 (2002).

[23] M. Telychko, P. Mutombo, M. Ondrcáček, P. Hapala, F. C. Bocquet, J. Kolorenč, M. Vondráček, P. Jelínek, and M. Švec, Achieving high-quality single-atom nitrogen doping of graphene/SiC $(0001)$ by ion implantation and subsequent thermal stabilization, ACS Nano 8, 7318 (2014).

[24] J. Sforzini, L. Nemec, T. Denig, B. Stadtmüller, T.-L. Lee, C. Kumpf, S. Soubatch, U. Starke, P. Rinke, V. Blum, F. C. Bocquet, and F. S. Tautz, Approaching Truly Freestanding Graphene: The Structure of Hydrogen-Intercalated Graphene on 6H-SiC(0001), Phys. Rev. Lett. 114, 106804 (2015)

[25] I. Gierz, C. Riedl, U. Starke, C. R. Ast, and K. Kern, Atomic Hole Doping of Graphene, Nano Lett. 8, 4603 (2008).

[26] J. P. Lewis, P. Jelínek, J. Ortega, A. A. Demkov, D. G. Trabada, B. Haycock, H. Wang, G. Adams, J. K. Tomfohr, 
E. Abad, H. Wang, and D. A. Drabold, Advances and applications in the FIREBALL ab initio tight-binding molecular-dynamics formalism, Phys. Status Solidi (b) 248, 1989 (2011).

[27] See the Supplemental Material of M. Švec, P. Hapala, M. Ondráček, P. Merino, M. Blanco-Rey, P. Mutombo,
M. Vondráček, Y. Polyak, V. Cháb, J. A. Martín Gago, and P. Jelínek, Silicene versus two-dimensional ordered silicide: Atomic and electronic structure of $\operatorname{Si}-(\sqrt{19} \times \sqrt{19}) R 23.4^{\circ} / \operatorname{Pt}(111)$, Phys. Rev. B 89, 201412(R) (2014). 